\title{
Evolución de la Productividad y el Costo Laboral en la Argentina Actual. Un Análisis Estilizado a Partir de las Cuentas Nacionales ${ }^{(*)}$
}

\author{
Evolution of Productivity and Labor Costs in Argentina Today. \\ An anAlysis STYLIZED from National ACCOUNTS
}

Pablo Manzanelli $i^{(*)}$

Resumen: Retomando las reflexiones de Ruy Mauro Marini en cuanto a ciertas peculiaridades que asume la apropiación del excedente en América Latina ("superexplotación del trabajo"), en este estudio se analizan las relaciones de distribución (productividad, costos salariales y beneficios) en la Argentina pre y pos devaluación de 2002. Todo ello es abordado con la finalidad de interpelar el papel que tiende a asumir el salario en la dinámica económica local. En tal sentido, se parte del supuesto de que la propia conformación estructural de la economía argentina y su forma de vinculación con el mercado internacional determinan que los salarios tiendan a adoptar la forma social de un costo de producción en lugar de un factor dinamizador de la demanda interna. No es la intención de este trabajo demostrar la validez (o no) de este interrogante, pero si la de aportar algunos elementos de juicio que contribuyan a interpretar algunas limitaciones que subyacen en el desarrollo económico actual de la Argentina.

Palabras clave: Distribución del ingreso — costos salariales — productividad — beneficios — tasa de ganancia.

\begin{abstract}
Going back to Ruy Mauro Marini's reflections referred to certain peculiarities that ownership of the surplus assumes in Latin America ("overexploitation of work"), this study explores the relations of distribution (productivity, salary costs and profit) in pre and post 2002-devaluation Argentina. This is addressed in order to question the role that wages tend to play in local economic dynamics. In this regard, it is assumed that the structural conformation of Argentina's economy itself and its way of relating with the international market determine that wages tend to adopt the social form of production cost rather than a domestic demand dynamizing factor. It isn't the intention of this work to demonstrate the validity (or not) of this question, but to provide some evidence to help interpret some limitations that underlie current economic development in Argentina.
\end{abstract}

Keywords: Distribution of income — wage costs — productivity — profit — rate of profit.

(*) Este trabajo se realizó en el marco del PICT-2008-0406: "La industria argentina en la posconvertibilidad: continuidades y rupturas en la dinámica y la estructura del sector", con patrocinio de la Agencia Nacional de Promoción Científica y Tecnológica. Se terminó de redactar el 08.09.2011.

(**) Licenciado en sociología de la Universidad de Buenos Aires e investigador del Área de Economía y Tecnología de la Facultad Latinoamericana de Ciencias Sociales (FLACSO), Sede Académica Argentina. E-mail: <pmanzanelli@flacso. org.ar>. Recebido em 13.09.11 e aceito em 15.12.11. 


\section{Presentación}

Diversas investigaciones han contribuido a determinar que tras la implosión del régimen de convertibilidad (1991-2001), se dio inicio a un nuevo ciclo de expansión centrado en los sectores productores de bienes transables, principalmente, de aquellos que se asientan en el aprovechamiento de las ventajas comparativas estáticas y/o institucionales de privilegio, desplazando, así, a la hegemonía de la valorización financiera como eje articulador del proceso de acumulación local (1976-2001) ${ }^{(1)}$.

Ello trajo aparejado, entre otras cosas, un elevado ritmo de crecimiento económico, una acelerada creación de puestos de trabajo y la consiguiente mejora - potenciada, a su vez, por un conjunto de políticas públicas - en diversos indicadores sociales. Sin embargo, las modalidades del patrón de especialización comercial de las ramas más dinámicas y su marcada orientación hacia los mercados externos, tienden a generar ciertas contradicciones de no menor importancia para el actual proceso económico.

A modo de hipótesis, se trataría, en este caso, del rol que tiende a asumir el salario en la dinámica económica. Es decir, la conformación estructural de la economía argentina y su forma de vinculación con el mercado internacional determinan que los salarios tiendan a adoptar la forma social de un costo de producción - que, como tal, debe ser ajustado al mínimo posible - en lugar de un factor dinamizador de la demanda interna y, por ende, impulsor del crecimiento económico.

A propósito, aunque se trate de un contexto local e internacional distinto, cabe traer a colación el concepto de "superexplotación del trabajo" de Ruy Mauro Marini, principalmente en lo que refiere a que entre los métodos de extracción del trabajo excedente $e^{(2)}$ (incremento de la tasa de explotación) se encuentre no sólo la utilización y/o ampliación de la capacidad productiva sino también, y fundamentalmente, la "superexplotación del trabajo", a través de: a) el aumento en la intensidad del trabajo, b) la prolongación de la jornada laboral, y c) la reducción del consumo del trabajador más allá de su límite normal (o sea, que parte del fondo necesario de consumo del trabajador se convierte en un modo específico para aumentar el tiempo de trabajo excedente).

(1) Cabe señalar que, profundizando las reformas estructurales (neoliberales) impulsadas a partir de la última dictadura militar (1976-1983), en el marco del decenio en el que estuvo vigente el régimen de la convertibilidad (conversión fija anclada al dólar estadounidense a la relación 1 a 1 durante 1991-2001) se implementaron las Leyes de Reforma del Estado (y posteriores privatizaciones) y de Emergencia Económica (remoción de ciertos subsidios, entre otros), se eliminaron (asimétricamente) una amplia gama de mecanismos regulatorios, se liberalizó el mercado cambiario y los flujos de divisas internacionales, se llevó a cabo la apertura externa de la economía, se "desreguló" el mercado de trabajo, etc. (Nochteff, 1999 o Basualdo, 2006). La salida de la convertibilidad, cristalizada en la sanción de la Ley nº 25.561 en los primeros días de 2002, derivó en un salto de significación del tipo de cambio que, de allí en más, tendió a ubicarse en niveles reales holgadamente superiores a los vigentes durante la convertibilidad, alterando los precios relativos y desplazando el eje de la acumulación, principalmente, hacia los sectores productores de bienes transables. A este período que se abre tras la megadevaluación de 2002 se denomina posconvertibilidad (Azpiazu y Schorr, 2010; CENDA, 2010 o KOSACOFF, 2008).

(2) Se entiende por trabajo excedente a la parte de la jornada laboral que se proyecta más allá de los límites del trabajo necesario (equivalente al valor de reproducción de la fuerza de trabajo), es decir, el tiempo de trabajo de la jornada laboral que si bien le cuesta gasto de fuerza de trabajo al trabajador no genera ningún valor para él (MARX, 1959). 
En sus palabras: "los tres mecanismos identificados - la intensificación del trabajo, la prolongación de la jornada de trabajo y la expropiación de parte del trabajo necesario al obrero para reponer su fuerza de trabajo - configuran un modo de producción fundado exclusivamente en la mayor explotación del trabajador, y no en el desarrollo de su capacidad productiva. Esto es congruente con el bajo nivel de desarrollo de las fuerzas productivas en la economía latinoamericana, pero también con los tipos de actividades que allí se realizan" (MARINI, 2007). Así, cabe remarcar que para este sociólogo y economista brasileño puede haber "superexplotación del trabajo" aun cuando los salarios se eleven, puesto que las exigencias para la reproducción de la fuerza de trabajo — vía un aumento en la intensidad de la tarea - pueden incrementarse en una proporción más elevada que los mismos, produciéndose un mayor desgaste físico e intelectual.

En efecto, más allá de que se trate de un escenario interno y mundial muy disímil ${ }^{(3)}$, es interesante interpelar a las relaciones económicas actuales desde esta perspectiva, y en virtud de ello a la conformación estructural de la economía argentina. En tal sentido, en tanto que las ramas más dinámicas se encuentren orientadas a abastecer de insumos (a través de la constelación de recursos naturales y/o de la exportación de commodities industriales) a las economías industrializadas, entonces la lógica de acumulación local se vuelve más dependiente - en términos relativos respecto a los otros factores de la producción - de la potenciación del trabajo y, por ende, del uso extensivo e intensivo de la fuerza de trabajo. Por consiguiente, en el marco de un significativo descenso de la tasa de desocupación y del impulso de diversas instancias de negociación sindicales, no parece casual que cobre mayor trascendencia que en otras etapas una intensa puja distributiva entre el capital y el trabajo.

Ello no se encuentra disociado de otro factor subyacente del actual proceso económico: la elevada concentración de la economía argentina y el sesgo del gran empresariado local hacia los mercados externos. A favor de la nueva paridad cambiaria, el mantenimiento de un "dólar alto", la vigencia de bajos costos salariales a escala mundial y un escenario internacional expansivo, las grandes empresas, insertas en los sectores productivos más favorecidos en la posconvertibilidad, se fortalecieron como sólidas plataformas productivas orientadas preferentemente hacia los mercados del exterior.

Al tiempo que tal proceso de concentración tiende a consolidar la conformación estructural del aparato productivo doméstico, profundiza las contradicciones en materia distributiva. Tal como lo sugiere las reflexiones de Kalecki (1956), cabe resaltar dos aspectos: 1) la participación de los salarios en el valor agregado está determinado por el grado de oligopolización de los mercados y la relación entre los precios de las materias primas y los costos del salario por unidad de producción; y 2) el aumento en el grado de oligopolio induce una redistribución del excedente de las empresas pequeñas a las grandes.

No es la intención de este trabajo demostrar la validez (o no) del conjunto de interrogantes aquí planteados, pero si la de aportar algunos elementos de juicio que contribuyan a interpretar algunos nudos problemáticos que, en este sentido, influyen en el devenir económico y social de la Argentina.

(3) Sobre la nueva y la vieja división internacional del trabajo se recomienda consultar Arceo (2005). 
De allí, que resulte relevante identificar y establecer un diagnóstico respecto al comportamiento de los principales indicadores que adquieren centralidad analítica a partir de la disputa abierta por la apropiación del trabajo excedente o, en otras palabras, por las relaciones de distribución que se desenvuelven en el ámbito de la producción; a saber: los costos salariales, la productividad del trabajo y la tasa de ganancia.

Con tales objetivos, en el presente ensayo se analiza, en primer lugar, la relación entre la productividad del trabajo y los costos salariales (visión estilizada de la tasa de explotación de la mano de obra) en la Argentina pre y pos devaluación de 2002. Como resultante de los comportamientos de tales variables, se indaga en la evolución de la participación de los beneficios y en la dinámica y el nivel de la tasa de ganancia apropiada por el conjunto del empresariado local.

En segundo término, y teniendo en cuenta el elevado grado de concentración de la estructura productiva local - con sus consecuencias en materia de apropiación de ganancias extraordinarias - , se pasa revista analítica al comportamiento de las relaciones de distribución (costos salariales, productividad y tasa de utilidades) teniendo como unidad analítica a las 500 firmas más grandes del país.

Siempre con el objeto de aportar algunos elementos de juicio en torno a las formas que adopta la puja distributiva en los últimos años, se presta particular atención a la identificación de continuidades y rupturas entre la posconvertibilidad y el período de vigencia del régimen de conversión fija con el dólar estadounidense.

\section{La apropiación del Excedente en el Conjunto de la Economía Argentina}

La estimación que aquí se desarrolla requiere de algunas aclaraciones metodológicas y/o conceptuales. La primera de ellas refiere a la necesidad de sustraer las amortizaciones de capital del producto bruto interno (PBI). Si se parte del supuesto de que el PBI se distribuye en salarios, beneficios y amortizaciones, la sustracción de estas últimas posibilita obtener el producto neto interno, que se distribuye entre el capital y el trabajo. Así, la delimitación de la participación de los salarios en ese producto neto consiste en dividir los salarios horarios reales (considerados como "costos laborales") por la productividad horaria del trabajo, multiplicando ese resultado — para mitigar el efecto que pudiera conllevar la utilización de distintos deflactores de precios — por la relación entre el índice de precios implícito del producto bruto y el índice de precios implícito del valor agregado neto. Como contraparte, se obtiene, en términos de beneficios y costos empresariales, la participación de los beneficios en el producto neto ${ }^{(4)}$.

La decisión metodológica de utilizar como deflactor al índice de precios implícito del producto bruto tiene como sustento analítico considerar al salario como un costo de producción. A su vez, la opción por calcular la productividad horaria del trabajo - en lugar de, como es habitual, usar como denominador la cantidad de ocupados - permite identificar el movimiento de la productividad sin contemplar a la prolongación de la

(4) Las metodologías de cálculo utilizadas en este apartado constituyen una adaptación de las formuladas por Glyn (et al., 1991). A su vez, no difieren conceptualmente de las empleadas por Duménil (et al., 2007) o Arceo (2009). 
jornada laboral como un factor determinante de la misma. Esto es particularmente importante porque, en el marco de la "flexibilización" de las relaciones laborales impulsada en los años noventa, el aumento de las horas trabajadas devino en un fenómeno bastante difundido. Por consiguiente, tal medición de la productividad horaria sólo puede incrementarse por la incorporación de tecnología, cambios en la organización del trabajo o, entre los principales factores, por un aumento en la intensidad del trabajo.

Por último, cabe aclarar que la ausencia de información básica que se extienda más allá de 2007 (particularmente de la cuenta de generación del ingreso del INDEC aunque, también, de la estimación del stock de capital fijo que elabora el mismo organismo) acota la dimensión temporal, para el agregado nacional, al período 1993-2007. No ocurre lo propio (aunque la forma de estimación es de un menor grado de sofisticación) en lo concerniente al análisis de las relaciones de distribución en las grandes empresas, cuyo tratamiento se aborda en la próxima sección.

A partir de estas breves aclaraciones, se presenta a continuación la evolución de la productividad del trabajo y los costos salariales y, a su vez, la relación entre ambos, esto es, una aproximación al grado de explotación de la fuerza de trabajo. Como puede advertirse en el Gráfico $\mathrm{n}^{\circ}$ 1, la misma guarda correspondencia con los avatares de la economía global, esto es, dos períodos marcadamente distintos en la década del noventa: incremento de la tasa de explotación en el período de auge y declinación en la etapa recesiva y de crisis final de la convertibilidad.

En el quinquenio 1993-1997, dicha tasa, que se incrementó 0,4 puntos porcentuales, conjugó incrementos de la productividad horaria $(13,3 \%)$ y una reducción del costo salarial $(7,7 \%)$, en el marco de una contracción del empleo y de un proceso de crecimiento económico. Es decir, en un contexto de aumento de la producción y de creciente sustitución de trabajo por capital, se aumentó en forma considerable la cantidad de bienes producidos y servicios suministrados por cada hora de trabajo, al tiempo que se abarató la remuneración promedio de esas horas de trabajo.

Luego, en el marco del período recesivo del régimen convertible (1998-2001), el descenso de 0,2 puntos porcentuales en el grado de explotación fue impulsado por la reducción de la productividad del trabajo $(-4,6 \%)$ o, más específicamente, porque el menor valor de los bienes y servicios producidos en esta etapa es mayor que la reducción de la cantidad de horas trabajadas. Lo que sugiere otra digresión: con una por demás elevada desocupación y teniendo en cuenta el proceso de flexibilización laboral, todo parecería indicar que una parte de ese aumento de la productividad fuera impulsada por la intensidad de la tarea desempeñada, siempre en el marco de una aguda caída de la actividad.

Por su parte, la formidable transferencia de ingresos que devino de la devaluación de la moneda provocó una reducción de 23,2\% del costo salarial por hora trabajada entre 2001 y 2003, que empujó a la tasa de explotación al alza, aun cuando la productividad del trabajo se encontraba en un ligero descenso. La ecuación esquemática sería la siguiente: menos producción por hora trabajada y, en un grado sumamente mayor, menor remuneración al trabajo por hora empleada, lo que da como resultante un excedente sobre el valor producido (recuérdese, del producto neto) mayor que la suma de salarios empleados como elementos productivos de ese proceso laboral. 
Gráfico n. 1. Argentina. Evolución de la productividad del trabajo, el costo salarial y la relación productividad/costo salarial, 1993-2007 (índice base 1993=100 y tasa)

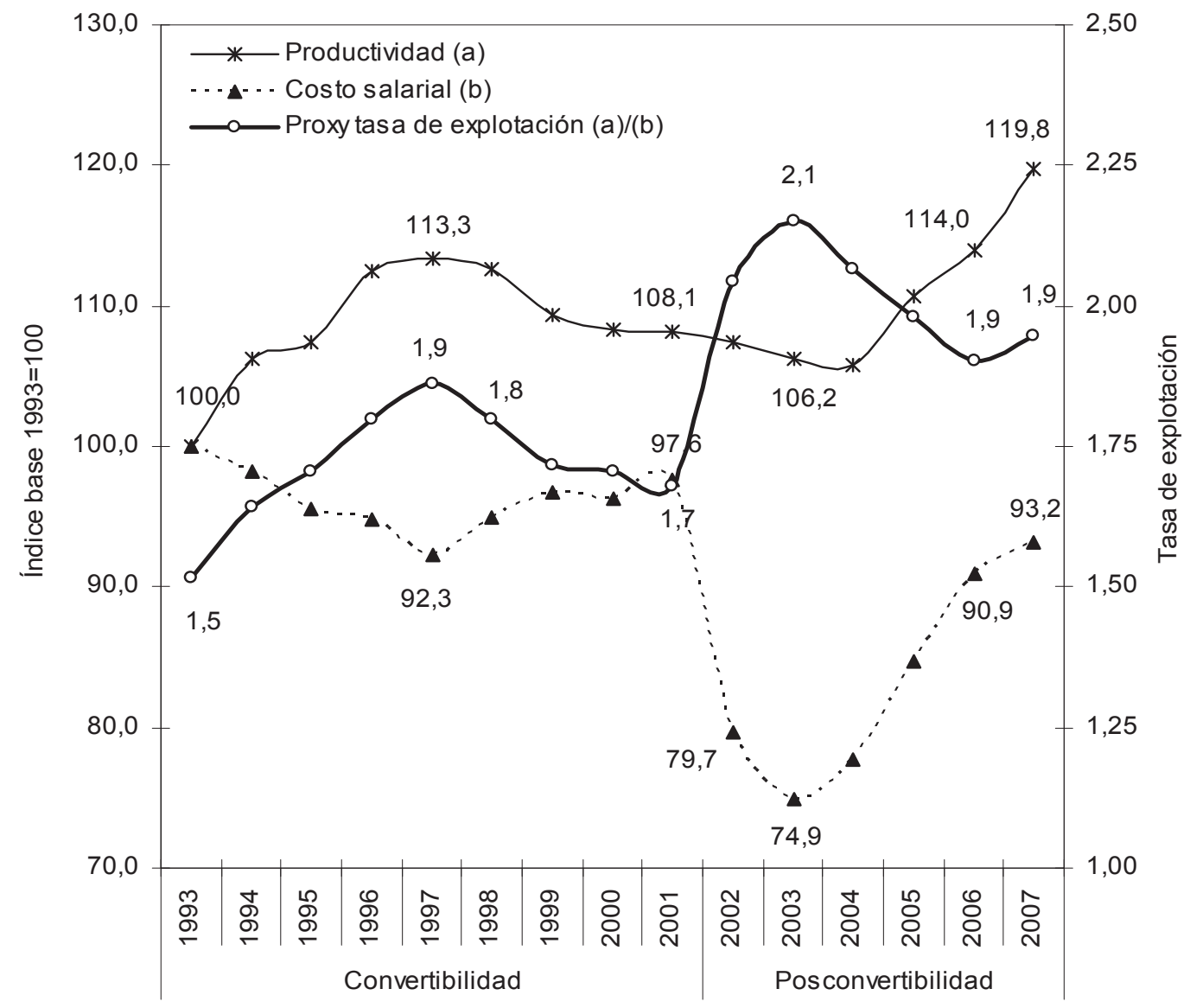

Fuente: Elaboración propia en base a información del INDEC.

Nota: La productividad del trabajo se estimó en base a la razón entre el valor agregado neto a precios constantes y la cantidad de horas trabajadas, mientras que el costo salarial corresponde a los salarios horarios reales (deflactados por el índice de precios implícito del producto bruto) y ajustados por el empleo por cuenta propia.

Posteriormente, durante el cuatrienio 2003-2006, si bien la recuperación del salario $(24,4 \%)$ redujo la más que importante tasa de explotación que tuvo como punto de partida, la mantuvo en un porcentaje elevado, el mismo que constituyera el pico más alto en la convertibilidad. Es decir, que si bien se pagó más por cada hora de trabajo en este período respecto a 2003, recién en 2007 se superó levemente la remuneración más baja de la convertibilidad, aunque el importante incremento de la productividad en este año no permitió disminuir (incluso lo acrecentó levemente) el trabajo excedente apropiado por el capital. 
En efecto, estas evidencias parecen indicar que la elevada tasa de explotación que se registra en la posconvertibilidad tuvo como principal factor explicativo al costo laboral en un nivel históricamente bajo. Ello no se encuentra disociado del desplazamiento de su función (cumplida en forma previa a la última dictadura militar, 1976-1983) como factor impulsor de la demanda a la de un simple "costo laboral" a escala mundial, al menos en las ramas más dinámicas del actual régimen económico. Lo que evidencia, primero, los legados críticos del modo de acumulación anterior (modelo de valorización financiera, 1976-2001) ${ }^{(5)}$, segundo, su profundización en la resolución de su crisis terminal (2001-2002) y, por último, la no reversión, a pesar de las altas tasas de crecimiento económico y del empleo, a sus niveles "históricos" durante el lustro posterior (2003-2007).

En estas condiciones, la maximización de la cuota de ganancia se apoya, principalmente, en la vigencia de un "dólar alto" y el mantenimiento de la remuneración al trabajo por debajo de su valor. Tal es así que en 2002, producto de la caída del salario real tras la depreciación cambiaria, el aumento de los beneficios ${ }^{(6)}$ operó como mecanismo principal de la recomposición de la tasa de ganancia (Gráficos nos 2 y 3), mientras que en los años posteriores la disminución relativa del trabajo excedente apropiado por el capital prácticamente no alcanzó a contraer dicha tasa, en el marco de un incremento absoluto de los beneficios, de la pervivencia de un costo salarial bajo en términos históricos y, entre otros factores que afectan a los beneficios relativos, de un tipo de cambio real elevado.

Tal es así, que la caída del costo salarial durante la megadevaluación en 2002 disparó, como factor más decisivo, la participación de los beneficios sobre el producto neto (Gráfico no 2). Esa situación excepcional, por el fuerte impacto inicial de la depreciación cambiaria, no fue revertida, en cuanto a sus niveles excepcionales, durante los años siguientes, aun cuando se haya percibido una, aun insuficiente, recuperación del atrasado costo salarial, así como una elevada generación de puestos de trabajo, en el marco de un importante ciclo ascendente de la economía doméstica.

(5) Sobre el modelo de valorización financiera, ver Basualdo (2006).

(6) Cabe una breve aclaración al respecto. A diferencia de las mediciones contables, la participación relativa de los beneficios consiste aquí, como fuera expuesto anteriormente, en identificar la parte que se apropian los capitalistas del valor neto producido y no de las ventas agregadas, es decir, del valor neto que se reparte entre el trabajo y el capital (o que se divide simplemente en salarios y beneficios). De allí que la recomposición de los beneficios en 2002 tan sólo quiera decir que el capital mejoró su situación relativa respecto al costo salarial pero no que haya recompuesto y abandonado su situación crítica en ese año. 
Gráfico n. 2. Argentina. Evolución de la participación de los beneficios en el producto neto, 1993-2007 (índice base 1993=100)

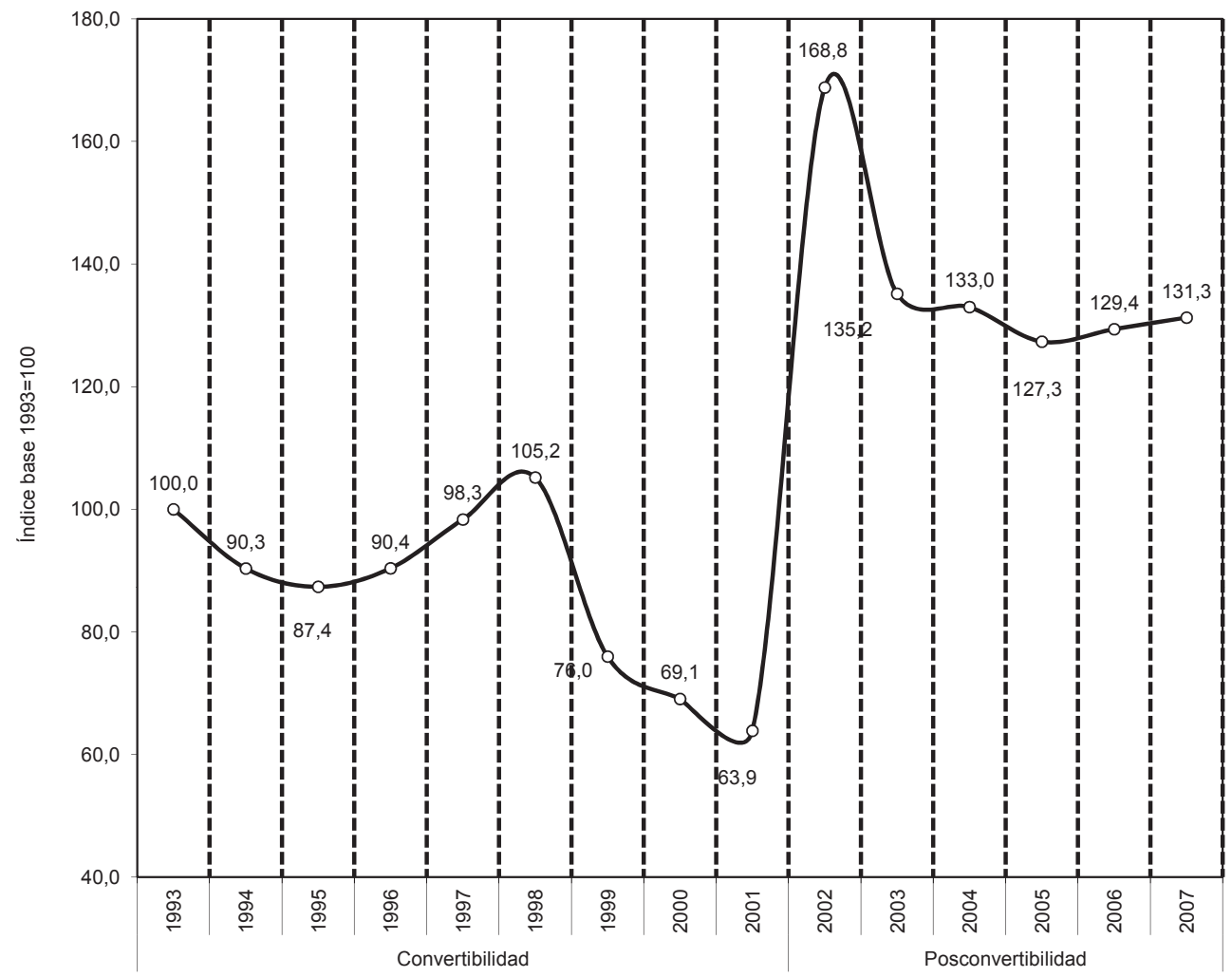

Fuente: Elaboración propia en base a información del INDEC.

Nota: La participación de los beneficios se obtuvo a partir de restar a la magnitud resultante de dividir los salarios horarios (deflactados por el índice de precios implícito del producto bruto) por la productividad horaria del trabajo y, a su vez, multiplicando ese resultado por la relación entre el índice de precios implícito del producto bruto y el índice de precios implícito del valor agregado neto.

De resultas de ello, y de un elevado excedente comercial $^{(7)}$, la gravitación de los beneficios en la posconvertibilidad fue más de un 50\% más elevada que la registrada durante el régimen convertible, y considerando los años extremos de la serie, la participación de los beneficios fue, en 2007, 31,3\% más alta que en $1993^{(8)}$.

(7) Siguiendo a Kalecki (1956) "las ganancias son iguales a la inversión más el excedente de exportaciones más el déficit presupuestal, menos el ahorro de los trabajadores, más el consumo de los capitalistas. En consecuencia un aumento del excedente de exportación aumentará las ganancias correspondientes siempre y cuando los otros componentes no se alteren... Los capitalistas de un país que consiga capturar los mercados extranjeros de otros países pueden aumentar sus ganancias a expensas de los capitalistas de otros países".

(8) En la historia reciente de la Argentina se pueden identificar cinco puntos de inflexión en cuanto a caídas significativas en la participación del salario en el ingreso: en 1976 esa gravitación se reduce en 13,6 puntos porcentuales, en 1982 cerca de 7 puntos, durante la crisis hiperinflacionaria de 1989 otros tantos no calculables, en 19965 puntos y en 2002 cerca de 7 puntos porcentuales (LINDEMBOIM et al., 2005). 
Este nuevo estadio en el nivel de la participación de los beneficios impulsa, como factor más decisivo, la tasa de ganancia al alza. Pero antes de indagar en su evolución y dinámica, cabe esbozar algunos comentarios introductorios sobre dicho indicador.

\section{RECUADRO N. 1. SÍNTESIS METODOLÓGICA DEL CÁLCULO DE LA TASA DE GANANCIA}

La tasa de ganancia fue calculada como la resultante de multiplicar la participación de los beneficios por la relación entre el producto neto y el stock de capital fijo. Al tiempo que la participación de los beneficios se obtuvo a partir de restar a la magnitud resultante de dividir los salarios horarios (deflactados por el índice de precios implícito del producto bruto) por la productividad horaria del trabajo y, a su vez, multiplicando el resultado por la relación entre el índice de precios del producto bruto y el índice de precios del valor agregado neto.

En efecto, las fórmulas del cálculo quedarían expresadas de la siguiente forma:

$$
P b=1-(w * 1 / L P * P q / P y)
$$

Siendo,

$\mathrm{Pb}$ : participación de los beneficios;

w: salario real por hora trabajada (donde el salario es ajustado por el empleo por cuenta propia);

$L P$ : productividad horaria laboral;

Pq: índice de precios del producto bruto; y

Py: índice de precios del valor agregado neto.

$$
r=P b *(N Y / N K)
$$

Donde:

$r$ : tasa de ganancia;

Pb: participación de los beneficios;

NY: valor agregado neto; $y$

NK: stock de capital (excluye stock de construcciones residenciales).

Esta metodología constituye una adaptación de la empleada en Glyn (et. al., 1991).

En su forma más general, el empresario pone en marcha un determinado negocio con el propósito de obtener un excedente sobre el monto invertido (salarios y capital comprometido). La tasa de ganancia es definida, en este sentido, como el ratio entre los beneficios obtenidos en un año y el capital total adelantado: capital constante más capital variable, teniendo en cuenta sus tasas de rotación. En la estimación que a continuación se presenta, debido a la falta de información que permita calcular la tasa de rotación del capital variable (salarios) y del restante capital circulante remite, en términos generales, al producto entre la participación de los beneficios y la relación entre el producto neto y el stock de capital fijo (edificios, maquinarias y equipos). En otras palabras, se mide el resultado medio de la suma total de capital fijo que debe invertir el conjunto del empresariado para obtener un determinado beneficio.

Al respecto, vale introducir una breve digresión que, aunque no invalida la relevancia de los resultados obtenidos, es importante tener en consideración. Se refiere a que la tasa de ganancia promedio de una economía tiene como limitante específico la 
imposibilidad de advertir las diferencias sectoriales y por rama de actividad en cuanto a sus niveles. Aun cuando la movilidad del capital y del trabajo tienden a igualar la tasa de ganancia en el largo plazo, la acotada dimensión histórico-temporal abordada y, a su vez, la constitución de oligopolios o cuasi oligopolios en distintos mercados, o bien, en el primer caso, restringe la posibilidad cierta de advertir esa movilidad, o, en el segundo, limita de hecho la misma, a punto tal de consolidar ganancias extraordinarias que tenderían a elevar la tasa de ganancia promedio. Si bien sobre este último punto se avanzará en la próxima sección, la falta de una serie de stock de capital fijo desagregado por ramas de actividad imposibilita la tarea de detectar las mencionadas heterogeneidades sectoriales.

Asimismo, por último, cabe señalar que, en cuanto al horizonte temporal, la ausencia, como se mencionó, de una serie de stock de capital que se extienda más allá de 2006 acota el cálculo de la tasa de ganancia al período 1993-2006. Si bien esta dimensión temporal omite un interregno de aumentos salariales, permite identificar el nivel y la dinámica de la tasa de ganancia con el mayor grado de sofisticación posible, así como evaluar su comportamiento durante el primer lustro de la posconvertibilidad.

A partir de estas aclaraciones, y tal como puede observarse en el Gráfico no 3, el análisis de la trayectoria de la tasa general de ganancia en la Argentina desde 1993 permite distinguir el auge y la decadencia de la convertibilidad y, posteriormente, los efectos inmediatos de la maxidevaluación (2002) y la consolidación del nuevo patrón de crecimiento a partir de 2003. Al respecto, se advierten sustanciales diferencias relativas en la tasa de ganancia de uno y otro período. Durante la vigencia del Plan de Convertibilidad la tasa de ganancia promedio alcanzó a 23,9\%, mientras que en el régimen de "dólar alto" dicha tasa trepó a $35,8 \%{ }^{(9)}$.

Así, las fluctuaciones de la tasa de ganancia durante el sexenio 1993-1998 son contemporáneas a los vaivenes del último ciclo ascendente del modelo de valorización financiera. En ese escenario, la elevada - aunque menor al del período posconvertibleparticipación de los beneficios y la relación relativamente constante entre el producto neto y el capital fijo determinaron un nivel de rentabilidad para nada desdeñable: $26,8 \%$.

(9) Estos niveles no parecen alejarse considerablemente de los estimados, con una metodología similar, por Michelena (2010). 
Gráfico n. 3. Argentina. Tasa general de ganancia: índice de rentabilidad sobre el capital fijo, 1993-2007 (porcentaje)

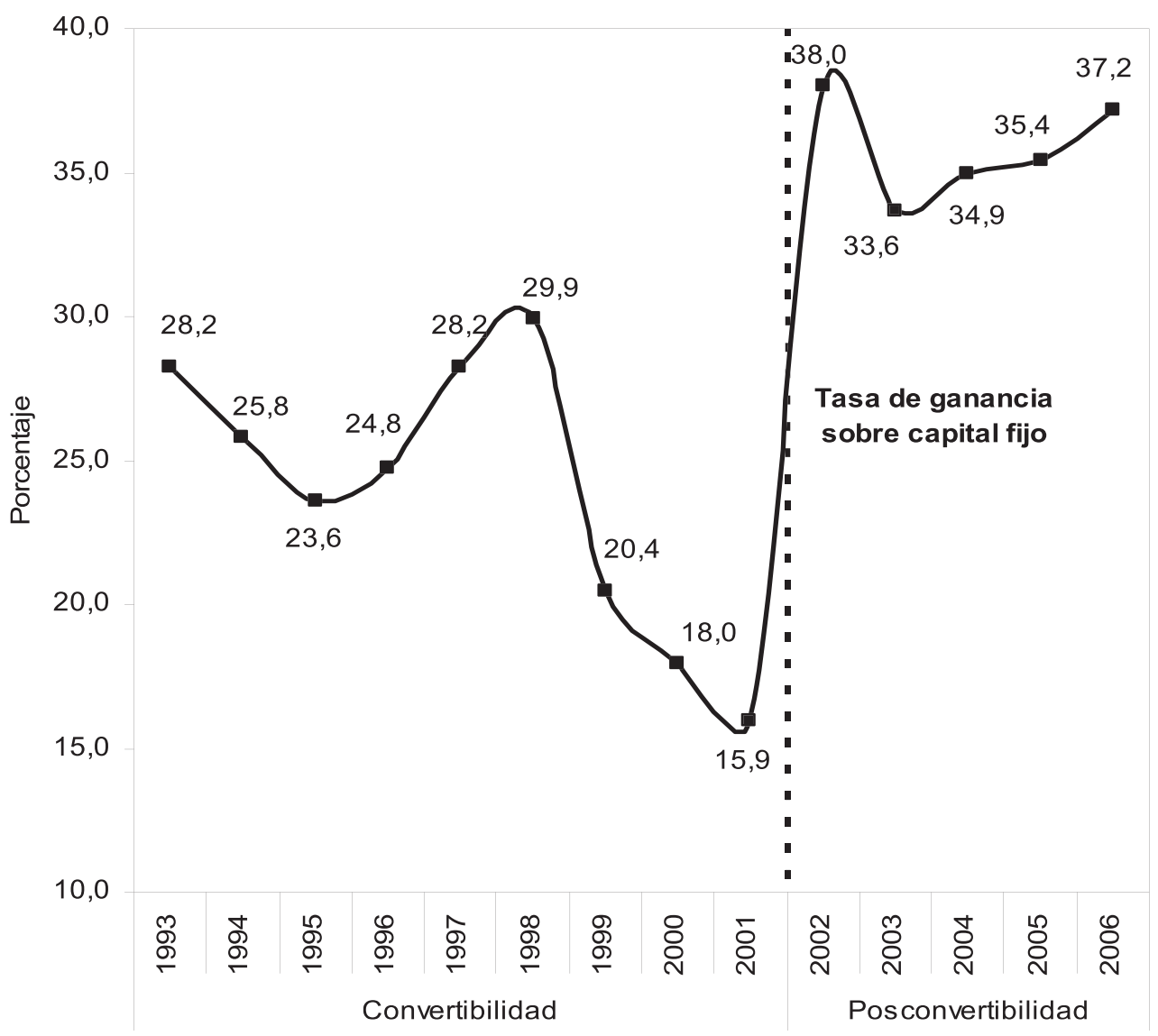

Fuente: Elaboración propia en base a información del INDEC.

Aunque resulte complejo determinar en qué medida la propia dinámica de la valorización financiera afectó el nivel de la tasa de ganancia, el predominio financiero, al desviar de la inversión productiva a los poseedores de capital, la apertura externa y desregulación asimétricas de los mercados — que abaratan relativamente los productos importados erosionando vía precios la producción interna — podrían haber afectado a la misma. Pero, fundamentalmente, cabe destacar que el impacto inicial del proceso de privatizaciones sobre la inversión fija, y su posterior tendencia descendente, expresó algunas de las limitaciones que asumió ese proceso de acumulación para incrementar la rentabilidad relativa del conjunto de la economía y, por ende, para potencialmente (aunque no necesariamente) expandir la producción global. De allí los tres años de contracción de la tasa de ganancia (1999-2001) y los cuatro de recesión económica (1999-2002), que constituyen unos de los principales sustratos del requerimiento de las fracciones dominantes para el cambio del modelo económico. 
Es que el descenso de la tasa de ganancia, como la lógica lo indica, perjudica el "normal" funcionamiento de la economía, reduciendo las posibilidades de dinamizar la acumulación de capital, o, dicho de manera simple, si bien las altas tasas de ganancia hacen que la inversión sea atractiva, la insuficiencia de las mismas provoca un freno a la inversión y, por ende, una reducción acumulativa de la producción. El juego de las interdependencias económicas adquiere una importancia fenomenal: el descenso de la inversión y la consecuente baja de la producción lleva a una menor demanda de bienes y de pago de salarios, reducciones que provocan, aun cuando no fuera un factor fundamental de la expansión, una disminución de la demanda interna en el marco de un acentuado déficit comercial y de restricción en el mercado de capitales. En efecto, el conjunto de estos comportamientos (y del agotamiento de las capacidades ociosas y las reformas estructurales) determinó, en la última etapa de la convertibilidad, la fase recesiva del ciclo económico, caracterizada por una más que elevada tasa de desocupación y por el descenso sistemático de la tasa de ganancia que experimentó una caída de 14 puntos porcentuales entre 1998 y 2001, hasta alcanzar en este último año su mínimo valor: 15,9\%.

Ahora bien, se podría sostener que, en la generalidad de los casos, la finalidad de los capitalistas consiste en maximizar su beneficio y, en un escenario competitivo, obtener ganancias extraordinarias a partir de la sustitución de trabajo por capital con el fin de alcanzar un precio de producción inferior al del mercado, el cual tendería, en dicho escenario, a ser adoptado por los restantes capitalistas. Es decir, que en el marco de la expansión global de la producción se desplazarían las innovaciones que permitieron tales ganancias extraordinarias a los capitales "menos eficientes". No obstante, en la Argentina de los años noventa, el régimen de acumulación y la orientación de las inversiones no determinó - por varios motivos, pero principalmente por la intensa centralización del capital - la igualación tecnológica y/u organizacional entre los diversos capitales. Las ganancias extraordinarias se apoyaron, en lo esencial, en la reducción del peso del costo salarial, en el marco de la hegemonía de la valorización financiera y el ajuste estructural.

El rol de la sobreexplotación del trabajo fue profundizado en la crisis de 2001-2002, a punto tal que el proceso devaluatorio implicó una caída del costo salarial de más del 23\%, empujando a la participación de los beneficios a su máximo "histórico" en 2002. Este componente de la rentabilidad es decisivo para interpretar el nivel de la tasa de ganancia en dicho año (38,0\%), mientras que el bajo costo laboral, aun en cierta recuperación, es un factor determinante para aprehender el sendero evolutivo que asumió el proceso de acumulación y sus inherentes contradicciones.

En forma posterior, ese bajo costo salarial asumió un papel central para mantener en un nivel alto a la participación de los beneficios en el producto neto y en virtud de ello, fundamentalmente, alcanzar una más que significativa tasa general de ganancia: $35,3 \%$, entre 2003 y 2006.

Teniendo en cuenta que la tasa de ganancia es la resultante de multiplicar la participación de los beneficios por el cociente entre la producción y el stock de capital ${ }^{(10)}$,

(10) Para facilitar la lectura de la relación producto/capital, Duménil y Lévy (2007) la denominaron "productividad del capital", aunque advierten que: "la noción de productividad del capital no subentiende que el capital cree valor en el sentido de la teoría marxista del valor". 
que, en última instancia, serían los beneficios obtenidos según el capital invertido, cabe introducir dos hipótesis interpretativas de sus resultados. Una es la que se vincula con la incidencia del primer componente, esto es, que en el marco de la sustantiva transferencia de ingresos del trabajo al capital, la más que importante participación de los beneficios asume un aspecto determinante para sostener la tasa de ganancia en niveles elevados. La otra guarda correspondencia con el segundo factor. Sobre el mismo, cabe apuntar que la considerable acumulación de capital durante la posconvertibilidad adquirió un papel no desdeñable en este proceso de crecimiento, cuya relación con el producto neto mostró un incremento de 7,8\% en ese quinquenio. En fin, como consecuencia de ambos procesos se tendió a sostener la curva ascendente de la (sumamente elevada) tasa de ganancia.

\section{Las Relaciones de Distribución en el Ámbito del Gran Empresariado Local}

La estructura económica argentina se caracteriza, en la actualidad, por una elevada concentración en la cúpula empresaria, tendencia que se ha profundizado en el marco de las transformaciones en el escenario macroeconómico de la posconvertibilidad ${ }^{(11)}$. Esto trajo aparejado consecuencias de diversa índole, por ejemplo, en materia de apropiación de ganancias extraordinarias que no se ven canalizadas a la formación de capital (AZPIAZU y MANZANELLI, 2011).

En lo que concierne a los efectos de la creciente concentración en materia de apropiación del trabajo excedente, cabe traer a colación una reveladora reflexión de Kalecki. En sus palabras: "la participación relativa de los salarios en el valor agregado en la industria la determinan, además de la estructura industrial del valor agregado, el grado de monopolio y la relación entre los precios de las materias primas y los costos-salario por unidad de producción. Si aumenta el grado de monopolio o se elevan los precios de las materias primas en relación con los salarios por unidad producida, la proporción representada por los salarios en el valor agregado disminuye" (KALECKI, 1956).

Pero también el grado de oligopolización de una economía tiene consecuencias en términos de distribución interempresarial del excedente, a punto tal que la concentración del capital persigue, como su proceso natural lo indica, la obtención de rentabilidades extraordinarias. Al decir de Kalecki: "Las variaciones del grado de monopolio no sólo tienen importancia decisiva en la distribución del ingreso entre trabajadores y capitalistas, sino en ciertos casos también, en la distribución del ingreso entre la propia clase capitalista. El aumento del grado de monopolio ocasionado por la expansión de las grandes empresas da lugar a que las industrias en que éstas predominan absorban una proporción mayor de los ingresos totales y las demás industrias una parte menor, es decir, el ingreso se redistribuye de las empresas pequeñas a las grandes".

En virtud de ello, y como se mencionó, de la fuerte concentración económica, resulta por demás interesante analizar las relaciones de distribución (o, más específicamente, el comportamiento de la productividad y el costo salarial) e identificar los niveles

(11) Entre otros puede consultarse, Azpiazu y Schorr (2010) para el sector industrial y PNUD (2009) para el conjunto de la economía. 
y comportamientos de la tasa de ganancia en el universo de las grandes empresas que se desempeñan en el ámbito local. Se trata de las 500 firmas de mayor tamaño del país que releva la Encuesta Nacional de Grandes Empresas (ENGE) que elabora el INDEC. Tal instrumento analítico es apropiado para los temas que aquí se estudian, puesto que brinda el resultado de un conjunto de variables (valor agregado bruto, amortizaciones, utilidades, salarios y contribuciones) que permiten — si bien con un menor grado de sofisticación que en el apartado anterior — un abordaje riguroso de la evolución de estos indicadores para el período 1993-2009, en el universo de las 500 compañías de mayor tamaño del país.

No obstante, cabe realizar algunas aclaraciones metodológicas respecto a ciertas diferencias subyacentes entre las estimaciones que a continuación se presentan y las que fueron analizadas, para el agregado nacional, en la sección anterior. Al respecto, al carecer de información respecto a la cantidad de horas trabajadas en la elite empresaria, se calculó la productividad del trabajo como el cociente entre el valor agregado neto en precios constantes (que surge de restar las amortizaciones al valor agregado bruto, y deflactar el resultado por el índice de precios mayoristas - IPIM) y el personal ocupado asalariado. Por su parte, el costo salarial resulta de dividir la sumatoria de los salarios y las contribuciones por la cantidad de asalariados, y deflacionar el resultado con el IPIM.

En base a estas consideraciones, los datos aportados por el Gráfico n. 4 son contundentes en indicar, primero, los elevados niveles de explotación del trabajo que se registraron en la elite empresaria durante la posconvertibilidad respecto a los vigentes durante los años noventa y, en segundo lugar, a pesar de que no sean estrictamente comparables entre sí, entre las 500 firmas de mayor tamaño y los que se advierten en el conjunto de la economía nacional.

Durante el régimen convertible puede advertirse que el costo laboral por ocupado se mantuvo constante, mientras que la productividad por ocupado trepó un 37,6\% entre 1993 y 2001. De resultas de ello, la tasa de explotación aumentó 0,5 puntos porcentuales, hasta alcanzar una tasa que implica que del producto neto generado por cada ocupado, más de la mitad (o alrededor del 60\%) se lo apropia el capital en razón de trabajo excedente. Del mismo modo que lo acontecido en el agregado nacional, aunque en un nivel mayor, tras la megadevaluación de 2002 se hundieron los costos salariales en un $38,6 \%$ en las grandes empresas.

Desde allí hasta 2007, se observan extraordinarias tasas de explotación: en ese sexenio el valor neto producido por cada trabajado cuadruplicó el trabajo necesario que obtuvo el trabajador en concepto de salario, o lo que es lo mismo, el peso del salario en el valor agregado neto fue de alrededor del 25\%, mientras que las tres cuartas partes restantes fueron internalizadas por el gran capital en concepto de trabajo excedente. Ello a pesar de la considerable recuperación del salario $(37,3 \%)$ que se vio levemente amortiguada por los incrementos de la productividad (21,9\%).

Si bien, es recién en el bienio posterior (2008-2009) donde la recuperación del costo salarial alcanza a superar los valores de 1993, y en 2009 los vigentes en 2001, lo cierto es que la tasa de explotación se mantuvo - incluso, a pesar de la desaceleración en el crecimiento de la productividad - en niveles por demás elevados, donde el valor neto de las grandes empresas fue de más de tres veces de lo que le cuesta cada trabajador. 
Gráfico n. 4. Argentina. Evolución de la productividad del trabajo, el costo salarial y la relación productividad/costo salarial en las 500 empresas más grandes del país, 1993-2009 (índice base $1993=100$ y tasa)

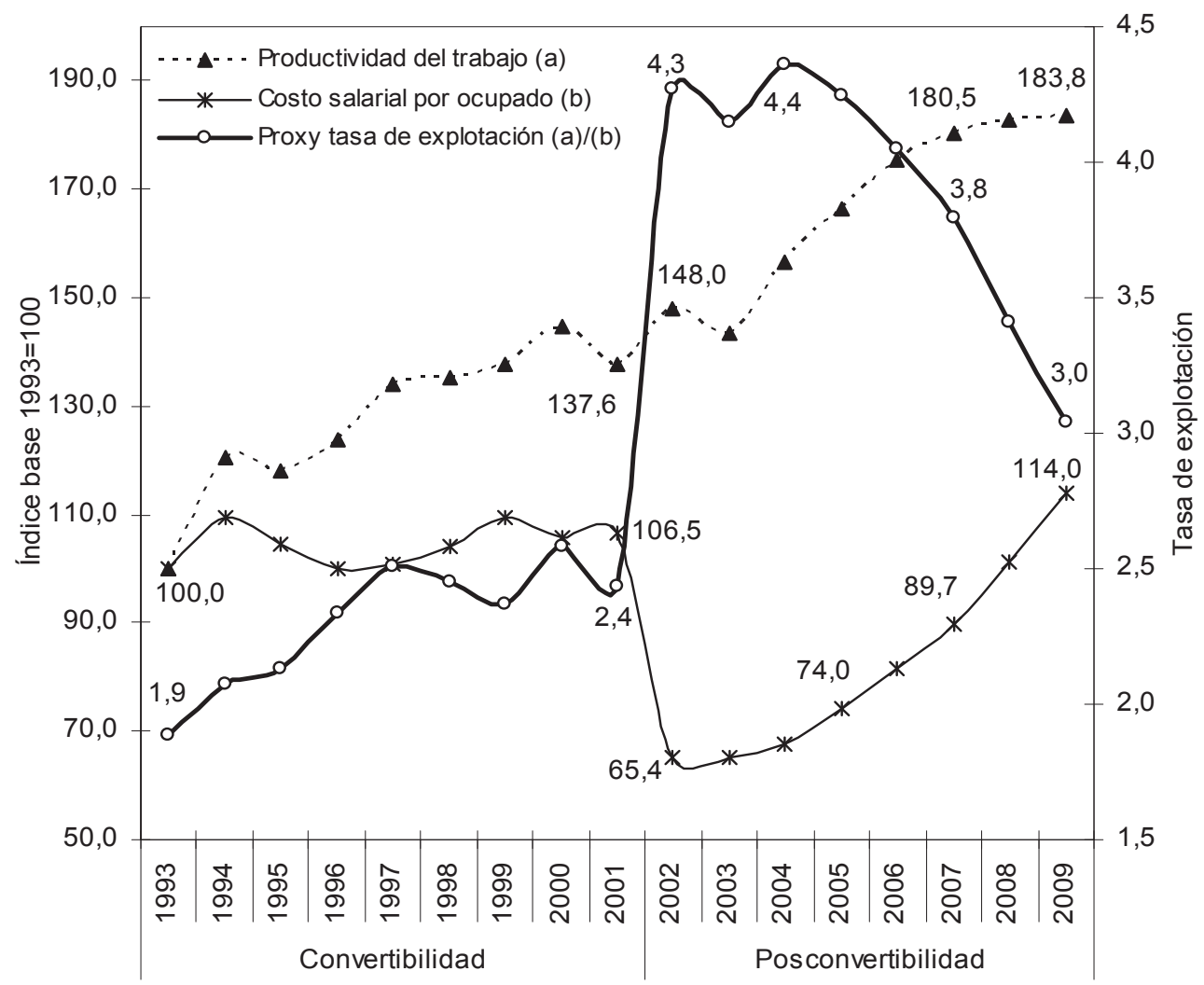

Fuente: Elaboración propia en base a información del INDEC.

Nota: La productividad del trabajo se estimó en base a la razón entre el valor agregado neto a precios constantes y la cantidad de personal ocupado asalariado, mientras que el costo salarial corresponde a los salarios reales (deflactados por el índice de precios del producto bruto) por ocupado.

Ello se ve reflejado, a su vez, de considerar la tasa de ganancia de este universo de grandes empresas. En este caso, dado que no se cuenta con información de stock de capital fijo, la estimación de este indicador surge de relacionar las utilidades netas (valor agregado neto menos salarios, contribuciones, indemnizaciones por despido, impuestos a la producción e intereses y rentas pagadas, y más subsidios corrientes e intereses y rentas cobradas) sobre el valor agregado neto. Se trata de una participación de los beneficios netos en el producto neto generado.

Lo primero que cabe señalar es, nuevamente, la diferencia que se advierte entre ambos regímenes económicos. Tal es así, que si bien la tasa de utilidades alcanzó un nivel para nada desdeñable durante la convertibilidad (23,4\%, entre 1993 y 2001), tras la maxidevaluación de 2002 la tasa de beneficios promedio trepó 13,1 puntos porcentuales en la posconvertibilidad (36,5\%, entre 2002 y 2009). 
Gráfico n. 5. Argentina. Tasa de utilidades netas sobre el valor agregado neto de las 500 empresas más grandes del país, 1993-2009 (porcentaje)

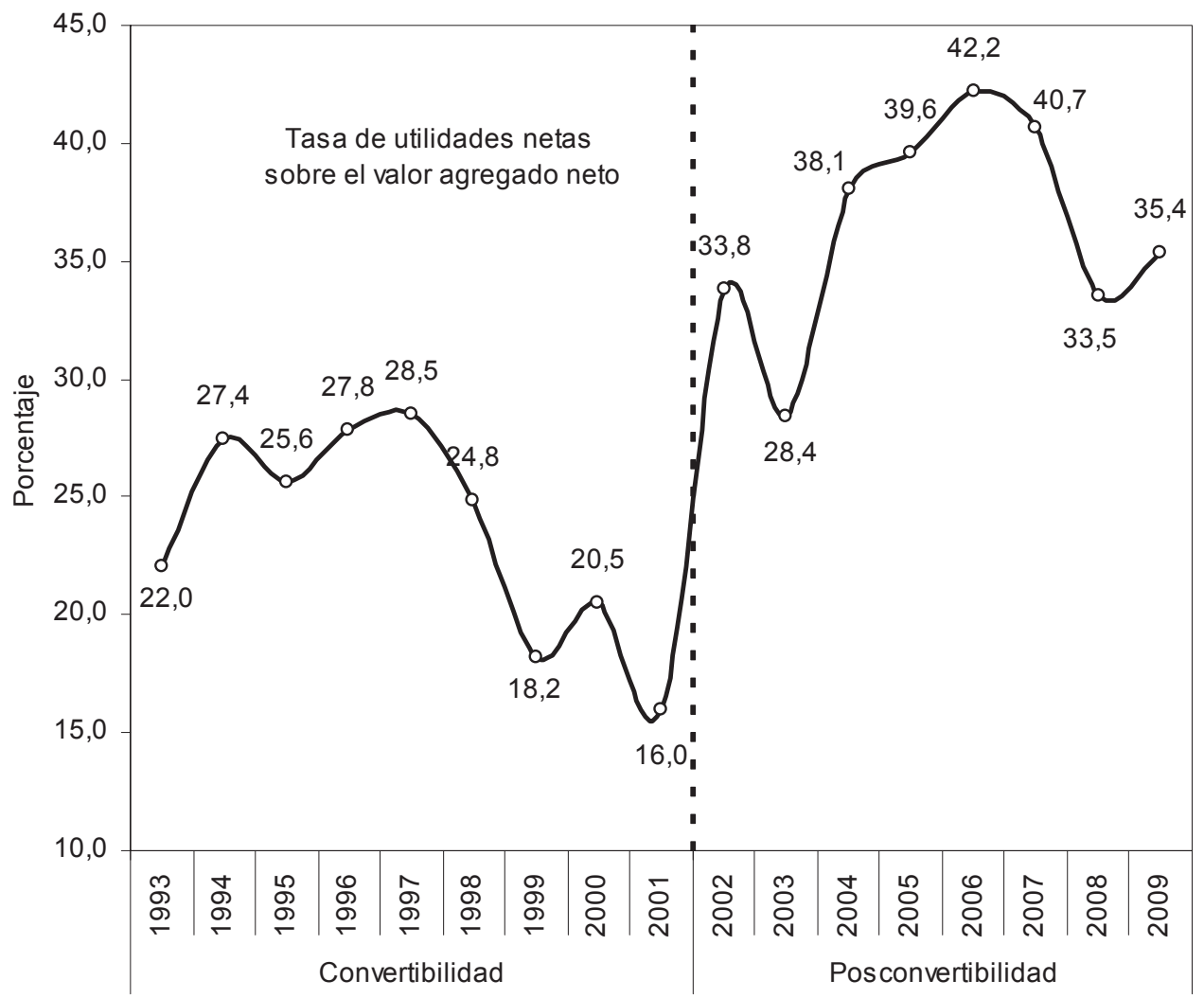

Fuente: Elaboración propia en base a información del INDEC.

En efecto, durante el transcurso del modelo que pivoteó en torno al "dólar alto", al tiempo que se registraron altas tasas de ganancia en el ámbito nacional y una elevada concentración económica de la estructura productiva local, las corporaciones que detentan el predominio económico del país - en este caso, las 500 firmas de mayor tamaño - internalizaron más que importantes cuotas de beneficio, holgadamente superiores a las que obtuvieron en los años noventa, crecientes durante el primer lustro (2002-2006, cuyo pico máximo fue de $42,2 \%$ en el último año) y ligeramente decrecientes, aunque en niveles por demás elevados, durante el último trienio (2007-2009) del régimen de la posconvertibilidad.

En este último punto, no deja de ser elocuente mencionar que, en el marco de la profunda crisis internacional de 2009, la tasa de utilidades netas se mantuvo en un nivel mucho más elevado que el de la convertibilidad y por demás atractiva para los capitales $(35,4 \%)$.

A juicio de Sylos Labini (1966): "Es correcto afirmar que los beneficios, superiores al mínimo, que reciben las empresas mayores tienen un carácter diferencial. Ahora bien, 
tales beneficios pueden admitirse también en el caso de competencia. Pero hay una diferencia sustancial entre los dos tipos de beneficio: los de competencia son debidos a 'disensiones' y son transitorios; los oligopolistas son debidos, en cambio, a características estructurales y son permanentes".

\section{Reflexiones Finales}

Los distintos análisis que integran este ensayo permiten concluir que en la posconvertibilidad ha tenido lugar un incremento significativo en el nivel de la explotación del trabajo, con su correlato en materia de apropiación de altas tasas de ganancia. En tal sentido, son varios los aspectos que interesa destacar:

- el costo laboral por hora trabajada cayó $13,2 \%$ en la posconvertibilidad respecto al período de vigencia del régimen de conversión fija con el dólar estadounidense, y la productividad horaria del trabajo aumentó menos del 2\% en el mismo período;

- la significativa caída del costo salarial en el marco de la megadevaluación de 2002, y su no reversión a sus niveles "históricos" durante el régimen de la posconvertibilidad, impulsó, como factor más determinante, una elevación en la tasa de explotación del trabajo y, como es lógico, de la participación de los beneficios en el producto neto: a) la tasa de explotación del trabajo de los años noventa se incrementó, en promedio, un $17,6 \%$ en el marco de la posconvertibilidad, y casi un 30\% entre 1993 y 2007 ; y b) la participación de los beneficios fue más de un $50 \%$ mayor en la posconvertibilidad que durante el régimen neoliberal;

- durante la década de 1990 la tasa de ganancia sobre el stock de capital alcanzó el 23,9\% mientras que durante el primer lustro del modelo de "dólar alto" dicha tasa trepó al 35,8\%;

- en el marco de un creciente proceso de concentración, el gran empresariado local incrementó las tasas de explotación del trabajo en casi el 70\% entre los períodos 1993-2001 y 2002-2009, a través de una reducción de más del 20\% del costo salarial y de un incremento del $30 \%$ en la productividad;

- las grandes empresas aumentaron sus tasas de utilidades netas sobre el valor agregado neto en 13 puntos porcentuales entre el régimen convertible y el que le sucedió tras la implosión del mismo en 2002.

En efecto, estos elementos parecen ser suficientes como para indicar que buena parte del incremento del excedente apropiado por el capital en el marco de la posconvertibilidad tuvo como sustento principal el incremento en la explotación del trabajo a través de una reducción en el costo salarial. Máxime si se considera que tal comportamiento tiene como punto de partida a un proceso de larga data (modelo de valorización financiera) que, en este sentido, parece haber impuesto, estructuralmente, un "techo salarial".

Si bien esto demandaría de nuevas investigaciones, este cuadro, de por sí complicado, resulta agravado porque las elevadas tasas de ganancia de la posconvertibilidad parecen haberse apropiado, en una medida no desdeñable, por el capital concentrado 
interno, acentuando la redistribución regresiva del ingreso y perpetuando, por su forma de inserción en la economía doméstica, al salario en un nivel histórica e internacionalmente bajo.

Dada la dependencia tecnológica, en la medida en que el proceso de reindustrialización no altere su subordinación a la producción y exportación de, principalmente, bienes primarios y manufacturados - que no forman parte esencial del consumo individual del trabajador — la forma específica que asumirá la maximización del excedente seguirá siendo la de presionar a la baja de los salarios. De tal manera, se tiende a reproducir la acumulación de capital basada en la superexplotación del trabajo, en dos sentidos: 1) porque el empresario local no tiene suficiente interés en incrementar la productividad para, haciendo bajar el valor por unidad de producto, depreciar la fuerza de trabajo, ya que lo producido internamente no determina, en lo fundamental, el valor de la fuerza de trabajo; y 2) porque la reducción del poder de compra de los trabajadores no le crea problemas significativos al capitalista en la esfera de la circulación, dado que no interfiere, en lo esencial, en la realización del producto, que se destina hacia los mercados internacionales y/o a los sectores de altos ingresos una vez consumada la, como afirma Marini, separación entre la esfera alta y la esfera baja de la circulación en el interior mismo de la economía.

En este contexto, uno de los nudos principales que debiera abordar un plan nacional de desarrollo con pretensiones de alcanzar un modelo de acumulación que priorice el desarrollo económico con inclusión social, es el de iniciar el dificultoso camino de desandar la forma en que se estructura la economía nacional, a partir del desarrollo de industrias más complejas, donde el proceso de producción se oriente, a partir del consumo interno de los sectores populares, a la esfera baja de la circulación.

\section{Bibliografía CitAdA}

ARCEO, Enrique. El impacto de la globalización en la periferia y las nuevas y viejas formas de dependencia en América Latina. Cuadernos del CENDES, Caracas, n. 60, 2005.

ARCEO, Enrique. El fin de un peculiar ciclo de expansión de la economía norteamericana. La crisis mundial y sus consecuencias. En: ARCEO, Enrique; BASUALDO, Eduardo y ARCEO Nicolás (Eds.). La crisis mundial y el conflicto del agro. Buenos Aires: UNQ, p. 12-CCC, 2009.

AZPIAZU, Daniel y MANZANELLI, Pablo. Reinversión de utilidades y formación de capital en un grupo selecto de grandes firmas (1998-2009). Realidad Económica, Buenos Aires, n. 257, 2011.

AZPIAZU, Daniel y SCHORR, Martín. Hecho en Argentina. Industria y economía, 1976-2007. Buenos Aires: Siglo Veintiuno Editores, 2010.

BASUALDO, Eeduardo. Estudios de historia económica. Desde mediados del siglo XX a la actualidad, Buenos Aires: Siglo XXI, 2006.

CENDA. La anatomía del nuevo patrón de crecimiento y la encrucijada actual. La economía argentina en el período 2002-2010. Buenos Aires: Cara o Ceca, 2010.

DUMÉNIL, Gérard y Lévy, Dominique. Crisis y salida de la crisis. Orden y desorden neoliberales. México D.F: Fondo de Cultura Económica, 2007.

GLYN, Andrew; HUGHES, Alan; LIPIETZ, Alan y SINGH, Ajit. The Rise and Fall of the Golden Age. En: MARGLIN, Steve y SCHOR, Juliet (Eds.). The Golden Age of Capitalism. Reinterpreting the Postwar Experience. Oxford: Clarendon Press, 1991. 
INDEC. Las grandes empresas en la Argentina, 1993-1997. Buenos Aires: 1999.

KALECKI, Michal. Teoría de la dinámica económica. Ensayo sobre los movimientos cíclicos y a largo plazo de la economía capitalista. México D. F: Fondo de Cultura Económica, 1956.

KOSACOFF, Bernardo. Crisis y recuperación y nuevos dilemas. La economía argentina 2002-2007. Buenos Aires: CEPAL, 2008.

LINDEMBOIM, Javier; GRAÑA, Juan y KENNEDY, Damián. Distribución funcional del ingreso en Argentina. Ayer y hoy. CEPED, Buenos Aires, n. 4, 2005.

MANZANELLI, Pablo. Evolución y dinámica de la tasa general de ganancia en la Argentina reciente. Realidad Económica, Buenos Aires, n. 256, 2010.

MARINI, Ruy Mauro. América Latina, dependencia y globalización. Buenos Aires: Clecço-Prometeo, 2007.

MARX, Karl. El capital. Crítica de la economía política. México D.F.: Fondo de Cultura Económica, 1959 .

MICHELENA, Gabriel. La evolución de la tasa de ganancia en la Argentina (1960-2007): caída y recuperación. Realidad Económica, Buenos Aires, n. 248, 2010.

NOCHTEFF, Hugo. La política económica en la Argentina de los noventa. Una mirada de conjunto. Revista Época: Buenos Aires, n. 1, 1999.

PNUD. Transformaciones estructurales de la economía argentina. Una aproximación a partir del panel de grandes empresas, 1991-2005. Buenos Aires, 2009.

SYLOS LABINI, Paolo. Oligopolio y progreso técnico. Barcelona: Ediciones Oikos-Tau, 1966. 